\title{
Efeito da tosquia na temperatura corpórea e outros parâmetros clínicos em ovinos ${ }^{1}$
}

\author{
Luiz Claudio N. Mendes ${ }^{2 *}$, Bruno H. Matsukuma², Gabriela de Oliveira², Livia C.T. Peres², \\ Bianca Gerardi ${ }^{2}$, Francisco Leydson F. Feitosa ${ }^{2}$, Sílvia H. Venturoli Perri ${ }^{3}$ e Juliana R. Peiró ${ }^{2}$
}

\begin{abstract}
Mendes L.C.N., Matsukuma B.H., Oliveira G., Peres L.C.T., Gerardi B., Feitosa F.L.F., Perri S.H.V. \& Peiró J.R. 2013. [Effect of shearing on the skin temperature and other clinical parameters in sheep.] Efeito da tosquia na temperatura corpórea e outros parâmetros clínicos em ovinos. Pesquisa Veterinária Brasileira 33(6):817-825. Departamento de Clínica, Cirurgia e Reprodução Animal, Faculdade de Medicina Veterinária, Universidade Estadual Paulista, Rua Clóvis Pestana 793, Araçatuba, SP 16050-680, Brazil. E-mail: Imendes@fmva.unesp.br

The aim of this study was to determine the variation of the temperature after shearing in sheep under dry and hot environment conditions and to compare the temperature changes with variation in cardiac and respiratory frequencies, ruminal movements and hydration status. Twenty Suffolk unshorn ewes were studied. Physical examination was performed in all animals three times a day at 7:00 AM, 1:00 PM and 7:00 PM, during 42 days (22 days before shearing and 20 days after shearing). The skin temperature was measured by infrared thermometer over several surfaces of the body. Data were submitted to analysis of variance, for comparisons between groups (shorn versus unshorn) at each time, and the significant difference was evaluated at level of $\mathrm{P}<0.05$ by Tukey test. The respiratory frequency was statistically significant at all times. When air humidity was high, the respiratory frequencies were low. The thermal stress was clear in sheep of this study, reflecting marked changes in cardiac and respiratory frequencies and rectal temperature. The respiratory frequency was the parameter more reliable to establish a framework of thermal stress in the unshorn sheep, with values on average three times higher than those reported in the literature. The heart rate monitors the thermal variation of the environment and is also an indicator of heat stress. This variation shows the Suffolk breed is well adapted to hot climates. The correlation between the body surface temperatures with environment temperature and air humidity was negative, as explained by the effect of wool insulation, i.e. even with an increase in environment temperature and humidity, the body temperature tends to maintain a compensating balance. In the shorn animals, the correlation between skin temperature with environment temperature and air humidity showed that the skin temperature increases when the environment temperature increases. The increase in the environment temperature does not affect the body temperature of unshorn animals due the insulating effect of the wool. However, when environment temperature rises, the presence of the wool starts to affect the thermal comfort as the heat absorption is larger than the capacity of heat loss. In this study, the best thermal stress indicators were the respiratory frequency and rectal and skin temperatures. The temperatures of the skin measured at the perineum, axillae and inner thigh were considered the most reliable.
\end{abstract}

INDEX TERMS: Sheep shearing, thermal stress, animal welfare.

\footnotetext{
${ }^{1}$ Recebido em 25 de fevereiro de 2013.

Aceito para a publicação em 22 de abril de 2013.

${ }^{2}$ Departamento de Clínica, Cirurgia e Reprodução Animal, Faculdade de Medicina Veterinária (FMVA), Universidade Estadual Paulista (Unesp), Rua Clóvis Pestana 793, Araçatuba, SP 16050-680, Brasil. *Autor para correspondência: Imendes@fmva.unesp.br

${ }^{3}$ Departamento de Apoio, Produção e Saúde Animal, FMVA-Unesp, Araçatuba, SP 16050-680, Brasil
}

RESUMO.- Os objetivos deste estudo foram determinar a variação da temperatura após a tosquia de ovinos em condição ambiental quente e seca e comparar as mudanças de temperatura com variações de frequências cardíaca e respiratória, movimentos ruminais e estado de hidratação. Vinte ovelhas Suffolk não tosquiadas foram estudadas. 0 exame físico foi realizado em todos os animais três vezes ao dia às 
7:00, 1:00 PM e 7:00 PM, durante 42 dias (22 dias antes do corte e 20 dias após o corte). A temperatura da superfície corporal foi medida pelo termómetro de infravermelho sobre vários pontos. Os dados foram submetidos à análise de variância, para comparações entre os grupos (tosquiado $\mathrm{x}$ não tosquiado) em cada tempo, e a diferença significativa foi avaliada em nível de $\mathrm{P}<0,05$ pelo teste de Tukey. A frequência respiratória foi estatisticamente significativa em todos os horários de cólera $(\mathrm{P}<0,05)$. Quando a umidade do ar estava alta, as freqüências respiratórias foram baixas $(\mathrm{P}=$ -1). 0 estresse térmico foi claro em ovinos deste estudo, refletindo mudanças acentuadas nas frequências cardíaca e respiratória e temperatura retal. A frequência respiratória foi o parâmetro mais confiável para estabelecer um quadro de estresse térmico em ovelhas não tosquiadas, com valores em média de três vezes maior do que os relatados na literatura. A correlação entre as temperaturas de superfície corpórea com a temperatura ambiente e umidade do ar foi negativa, explicado pelo efeito de isolamento de lã, ou seja, mesmo com um aumento da temperatura ambiente e umidade, a temperatura do corpo tende a manter um equilíbrio de compensação. Nos animais tosquiados, a correlação entre a temperatura da pele com a temperatura ambiente e umidade do ar mostraram que a temperatura da pele aumenta quando aumenta a temperatura ambiente. 0 aumento da temperatura ambiente não afeta a temperatura do corpo dos animais não tosquiados devido ao efeito isolante da lã. No entanto, quando a temperatura ambiente sobe, a presença da lã começa a afetar o conforto térmico, já que a presença desta dificulta a perda de calor pela sudação. Neste estudo, os melhores indicadores de estresse térmico foram a frequência respiratória e temperaturas retais e da pele. As temperaturas da pele medidas na face interna da coxa, axilas e períneo foram consideradas as mais confiáveis.

TERMOS DE INDEXAÇÃO: Ovinos, tosquia, estresse térmico, bem estar animal.

\section{INTRODUÇÃO}

Animais nos trópicos sempre apresentam produção em níveis inferiores, se comparados àqueles criados em clima temperado (Quesada et al. 2001). As limitações à produção em áreas tropicais podem ser ocasionadas pelos quatro principais elementos ambientais estressantes: temperatura do ar, umidade do ar, radiação do sol e velocidade do vento (Quesada et al. 2001). Estudos desenvolvidos por Hopkins et al. (1978) e Marai et al. (2008) demonstraram que existem alterações tanto na produção como na reprodução dos ovinos, face às alterações das condições ambientais. Já nos animais domésticos, a temperatura corporal apresenta períodos de oscilação espontâneos e regulares em diferentes épocas do ano, e o resultado desse complexo mecanismo é prova da existência de fatores endógenos e exógenos (Piccione et al. 2002).

A lã dos ovinos tem a capacidade isolante térmica conhecida, e são conhecidos também os meios pelos quais os animais homeotérmicos mantêm sua temperatura, proporcionando um equilíbrio térmico. Nos ambientes tropicais, a temperatura do ar tende a ser próxima da ou maior que a corporal, o que torna ineficazes os mecanismos de transferência térmica por condução e convecção (Silva et al. 2003), sobrando a função de termorregulação para evaporação, pois as trocas passam a não depender do diferencial de temperatura entre o organismo e a atmosfera. Nos ruminantes o equilíbrio térmico é alcançado principalmente pela perda evaporativa de calor através das vias aéreas e pela secreção sudorípara que, segundo Anderson (1996), pode chegar a $150 \mathrm{~g} / \mathrm{m}^{2} / \mathrm{h}$ a $40^{\circ} \mathrm{C}$ nos bovinos e a $32 \mathrm{~g} / \mathrm{m}^{2} / \mathrm{h}$ durante o estresse calórico em ovinos tosquiados, mostrando que a secreção de suor nos ovinos é menos importante que na vaca.

O rebanho lanado e com aptidão para produção de carne Vem se desenvolvendo nas regiões Sudeste e Centro-Oeste, e os locais de maior concentração do rebanho são os locais onde as temperaturas médias são as mais altas, justificando a preocupação com o estresse térmico desses animais (Matsukuma et al. 2010).

A tosquia é a retirada da lã, realizada em ovinos lanados, com aptidão para produção de lã ou para produção de carne, e pode ser feita manual ou mecanicamente. Nas raças com aptidão para carne a lã é um produto secundário, mas a tosquia é prática indispensável. É realizada normalmente uma vez ao ano, variado de acordo com a raça e com o clima da região. No Brasil se aplica geralmente nos meses de outubro, novembro e dezembro. Também é utilizada como medida higiênico-sanitária para as ovelhas prenhes, para machos em estação de monta e como manejo que visa aumentar a ingestão de alimentos nas fêmeas pré cobertura (Carvalho et al. 2004).

A tosquia causa uma marcada, porém transitória, elevação na temperatura retal (Hopkins et al. 1978), de mais de $1^{\circ} \mathrm{C}$ na temperatura corporal, e essa elevação de temperatura é explicada pela reação contrária promovida pelo estresse da perda da cobertura de lã. Resultados obtidos mostram a perda transitória no ritmo da temperatura corporal pela tosquia, com um componente exógeno, a tosquia em si, e um componente endógeno, as modificações dos níveis metabólicos induzidos pela remoção da lã, a camada isolante externa (Piccione et al. 2002). Já Aleksiev et al. (2003) demonstraram por meio de dois grupos de ovelhas, um recebendo água ad libitum e outro com privação hídrica, a não significativa alteração na temperatura retal pós-tosquia.

A hipertermia pós-tosquia promove um aumento no metabolismo normal dos animais que consequentemente aumenta o consumo de alimentos. Este fato foi observado no estudo de Çam et al. (2007), onde foi observado um maior consumo de alimentos e um melhor ganho de peso, assim como um melhor acabamento de carcaça, em cordeiros machos em idade de abate, em comparação com cordeiros machos não tosquiados. Uma das hipóteses lançadas é a compensação térmica com produção de camada de tecido adiposo isolante. A tosquia tende a aumentar o consumo alimentar em até $50 \%$, ocasionando-se uma alteração no tempo de descanso entre os pastejos (Filho et al. 2001). Esta elevação de temperatura pós-tosquia pode durar semanas diferentemente da hipertermia induzida pelo 
estresse, onde o aumento de temperatura dura somente horas (Piccione et al. 2002).

São poucos os estudos que relatam a hipertermia pós-tosquia dos ovinos, e são mais infrequentes os que descrevem esse fenômeno, e são direcionados ao estresse calórico, sendo conduzidos na Austrália e nos Estados Unidos (Quesada et al. 2001). Mensurações realizadas após a tosquia, em raças mediterrâneas na Itália, onde a temperatura ambiente variou de 16 a $21^{\circ} \mathrm{C}$, mostraram que a remoção da camada de lã determina uma alteração na homeostase térmica de mais de $1^{\circ} \mathrm{C}$ na temperatura retal 30 dias pós-tosquia, provavelmente pelo fator adaptação ao ambiente, considerando a zona termoneutra dos ovinos tosquiados de $28^{\circ} \mathrm{C}$, os autores chegaram a conclusão que os animais sofreram estresse térmico pelo frio (Piccione et al. 2003).

0 estudo abaixo descrito objetivou verificar a variação da temperatura corpórea pós-tosquia em ovinos criados em clima quente e seco, e de se correlacionar as variações da temperatura retal, da pele, e também as freqüências cardíaca e respiratória, movimento ruminal e estado de hidratação com a umidade e temperatura ambiental.

\section{MATERIAL E MÉTODOS}

Foram utilizadas 20 ovelhas da raça Suffolk, com três anos (média) com a lã íntegra de aproximadamente cinco centímetros, mantidas em curral com sombreamento parcial, suplementadas com ração total ${ }^{4}$ e água ad libitum.

Os sete primeiros dias foram reservados para o período de adaptação dos animais ao ambiente. 0 curral, que possuía área coberta, também era equipado com três cochos e bebedouros suficientes para os 20 animais, tronco de contenção e brete, próprios para ovinos, localizados na área com cobertura. 0 período experimental teve duração de 42 dias, sendo os primeiros 22 dias reservados para a avaliação dos parâmetros clínicos dos animais antes da tosquia experimento, e os 20 últimos dias foram reservados para a coleta dos dados dos animais após a tosquia.

Os animais foram examinados diariamente, três vezes ao dia, às 7,13 e 19 horas, durante os 42 dias, 22 dias antes da tosquia e 20 pós-tosquia, os animais foram examinados rigidamente da mesma forma nos dois momentos.

Foram mensuradas, em todos os animais, as frequências cardíaca e respiratória, movimento ruminal, estado de hidratação, temperaturas retal e da superfície corpórea e outras observações quando necessárias. As freqüências e o movimento ruminal foram aferidos com auxilio de estetoscópios, já a hidratação era conferida a partir da observação da elasticidade da pele (locais sem lã) e umidade da mucosa oral. Estes dados foram anotados em fichas clínicas e posteriormente analisados estatisticamente.

A temperatura ambiente e a umidade relativa do ar foram obtidas pelo termo higrômetro ${ }^{5}$ e anotadas em todos os horários em todos os períodos do dia (Quadros 1 e 2). A temperatura retal foi aferida por termômetro de mercúrio. A temperatura da pele foi mensurada através de termômetro infravermelho de mão e de não contato ${ }^{6}$. A temperatura da pele teve suas medidas definidas nas regiões da fronte (TF), nuca externa e interna a lã (TIN e TEN), dorso externo e interno a lã (TED e TID), axilas (TA), face interna (TFIC) e externa das coxas externa e interna a lã (TEFEC e TIFEC) e períneo (TP), no máximo, a dez centímetros de distân-

\footnotetext{
${ }^{4} \mathrm{LB}^{\circledR}$ - Araçatuba, SP.

${ }^{5}$ Hygrotherm TH02 - IMPAC, São Paulo, Brasil.

${ }^{6}$ Fluke 66 - Fluke Corporation, USA.
}

Quadro 1. Representação das temperaturas ambiente $\left({ }^{\circ} \mathrm{C}\right)$ e umidade do ar nos momentos antes da tosquia representados pelos dias verticalmente e subdivididos em seus períodos manhã, tarde e noite

\begin{tabular}{|c|c|c|c|c|c|}
\hline \multicolumn{3}{|c|}{ Não Tosquiado } & \multicolumn{3}{|c|}{ Não Tosquiado } \\
\hline \multicolumn{3}{|c|}{ Temperatura ambiente } & \multicolumn{3}{|c|}{ Umidade ar } \\
\hline \multirow[t]{3}{*}{ D1 } & Manhã & 21,9 & D1 & Manhã & 74 \\
\hline & Tarde & 27,8 & & Tarde & 58 \\
\hline & Noite & 22,3 & & Noite & 76 \\
\hline \multirow[t]{3}{*}{ D2 } & Manhã & 20,9 & D2 & Manhã & 53 \\
\hline & Tarde & 32 & & Tarde & 41 \\
\hline & Noite & 29,6 & & Noite & 46 \\
\hline \multirow[t]{3}{*}{ D3 } & Manhã & 23,2 & D3 & Manhã & 67 \\
\hline & Tarde & 35 & & Tarde & 27 \\
\hline & Noite & 31 & & Noite & 37 \\
\hline \multirow[t]{3}{*}{ D4 } & Manhã & 23,2 & D4 & Manhã & 60 \\
\hline & Tarde & 36,4 & & Tarde & 26 \\
\hline & Noite & 33 & & Noite & 31 \\
\hline \multirow[t]{3}{*}{ D5 } & Manhã & 24,7 & D5 & Manhã & 49 \\
\hline & Tarde & 36,9 & & Tarde & 24 \\
\hline & Noite & 32,9 & & Noite & 29 \\
\hline \multirow[t]{3}{*}{ D6 } & Manhã & 23,6 & D6 & Manhã & 66 \\
\hline & Tarde & 37 & & Tarde & 29 \\
\hline & Noite & 32,7 & & Noite & 32 \\
\hline \multirow[t]{3}{*}{ D7 } & Manhã & 42 & D7 & Manhã & 46 \\
\hline & Tarde & 32,1 & & Tarde & 35 \\
\hline & Noite & 36,8 & & Noite & 38 \\
\hline \multirow[t]{3}{*}{ D8 } & Manhã & 52,6 & D8 & Manhã & 53 \\
\hline & Tarde & 46,6 & & Tarde & 41 \\
\hline & Noite & 43,5 & & Noite & 50 \\
\hline D9 & Manhã & nc & D9 & Manhã & nc \\
\hline & Tarde & $\mathrm{nc}$ & & Tarde & nc \\
\hline & Noite & nc & & Noite & nc \\
\hline D10 & Manhã & 49,8 & D10 & Manhã & 56 \\
\hline & Tarde & 45,7 & & Tarde & 43 \\
\hline & Noite & 40,7 & & Noite & 45 \\
\hline D11 & Manhã & 46,8 & D11 & Manhã & 53 \\
\hline & Tarde & 50,1 & & Tarde & 48 \\
\hline & Noite & 41,6 & & Noite & 47 \\
\hline D12 & Manhã & 46,7 & D12 & Manhã & 55 \\
\hline & Tarde & 41,8 & & Tarde & 40 \\
\hline & Noite & 39 & & Noite & 40 \\
\hline D13 & Manhã & 42,6 & D13 & Manhã & 52 \\
\hline & Tarde & 40,5 & & Tarde & 34 \\
\hline & Noite & 41,3 & & Noite & 37 \\
\hline D14 & Manhã & 41,4 & D14 & Manhã & 44 \\
\hline & Tarde & 34,2 & & Tarde & 35 \\
\hline & Noite & 33,5 & & Noite & 36 \\
\hline D15 & Manhã & 41,9 & D15 & Manhã & 46 \\
\hline & Tarde & 35,4 & & Tarde & 31 \\
\hline & Noite & 31,6 & & Noite & 32 \\
\hline D16 & Manhã & 33 & D16 & Manhã & 46 \\
\hline & Tarde & 40,5 & & Tarde & 34 \\
\hline & Noite & 35,8 & & Noite & 41 \\
\hline D17 & Manhã & 42 & D17 & Manhã & 45 \\
\hline & Tarde & 37,5 & & Tarde & 33 \\
\hline & Noite & 36,3 & & Noite & 39 \\
\hline D18 & Manhã & 44,7 & D18 & Manhã & 51 \\
\hline & Tarde & 44,1 & & Tarde & 39 \\
\hline & Noite & 42,3 & & Noite & 42 \\
\hline D19 & Manhã & 45,9 & D19 & Manhã & 49 \\
\hline & Tarde & 43,2 & & Tarde & 36 \\
\hline & Noite & 49,3 & & Noite & 46 \\
\hline D20 & Manhã & 51,1 & D20 & Manhã & 56 \\
\hline & Tarde & 50,9 & & Tarde & 48 \\
\hline & Noite & 49,3 & & Noite & 51 \\
\hline D21 & Manhã & 47,9 & D21 & Manhã & 53 \\
\hline & Tarde & 49,6 & & Tarde & 43 \\
\hline & Noite & 39,1 & & Noite & 41 \\
\hline D22 & Manhã & 45,7 & D22 & Manhã & 49 \\
\hline & Tarde & 42,4 & & Tarde & 41 \\
\hline & Noite & & & Noite & \\
\hline & não cole & & & não cole & \\
\hline
\end{tabular}

Pesq. Vet. Bras. 33(6):817-825, junho 2013 
Quadro 2. Representação das temperaturas ambiente $\left({ }^{\circ} \mathrm{C}\right) \mathrm{e}$ umidade do ar nos momentos após a tosquia representados pelos dias verticalmente e subdivididos em seus períodos manhã, tarde e noite

\begin{tabular}{|c|c|c|c|c|c|}
\hline \multicolumn{3}{|c|}{ Tosquiado } & \multicolumn{3}{|c|}{ Tosquiado } \\
\hline \multicolumn{3}{|c|}{ Temperatura ambiente } & \multicolumn{3}{|c|}{ Umidade ar } \\
\hline \multirow[t]{3}{*}{ D1 } & Manhã & & D1 & Manhã & \\
\hline & Tarde & & & Tarde & \\
\hline & Noite & 28,4 & & Noite & 65 \\
\hline \multirow[t]{3}{*}{ D2 } & Manhã & 24,3 & D2 & Manhã & 84 \\
\hline & Tarde & 23,9 & & Tarde & 93 \\
\hline & Noite & 24,7 & & Noite & 93 \\
\hline \multirow[t]{3}{*}{ D3 } & Manhã & 20,5 & D3 & Manhã & 95 \\
\hline & Tarde & 29,7 & & Tarde & 62 \\
\hline & Noite & 29,8 & & Noite & 61 \\
\hline \multirow[t]{3}{*}{ D4 } & Manhã & 24,9 & D4 & Manhã & 80 \\
\hline & Tarde & 33,8 & & Tarde & 51 \\
\hline & Noite & 22,8 & & Noite & 90 \\
\hline \multirow[t]{3}{*}{ D5 } & Manhã & 22,2 & D5 & Manhã & 86 \\
\hline & Tarde & 29,5 & & Tarde & 63 \\
\hline & Noite & 33,3 & & Noite & 49 \\
\hline \multirow[t]{3}{*}{ D6 } & Manhã & 23,2 & D6 & Manhã & 83 \\
\hline & Tarde & 33,2 & & Tarde & 54 \\
\hline & Noite & nc & & Noite & nc \\
\hline \multirow[t]{3}{*}{ D7 } & Manhã & 21,6 & D7 & Manhã & 89 \\
\hline & Tarde & 28,6 & & Tarde & 65 \\
\hline & Noite & 28 & & Noite & 67 \\
\hline \multirow[t]{3}{*}{ D8 } & Manhã & 23,9 & D8 & Manhã & 75 \\
\hline & Tarde & 29,8 & & Tarde & 61 \\
\hline & Noite & 30,4 & & Noite & 63 \\
\hline \multirow[t]{3}{*}{ D9 } & Manhã & 25 & D9 & Manhã & 79 \\
\hline & Tarde & 31,7 & & Tarde & 60 \\
\hline & Noite & 24,1 & & Noite & 73 \\
\hline \multirow[t]{3}{*}{ D10 } & Manhã & 22,1 & D10 & Manhã & 83 \\
\hline & Tarde & 30,1 & & Tarde & 59 \\
\hline & Noite & 33,7 & & Noite & 44 \\
\hline \multirow[t]{3}{*}{ D11 } & Manhã & 23,3 & D11 & Manhã & 82 \\
\hline & Tarde & 27,8 & & Tarde & 71 \\
\hline & Noite & 31,2 & & Noite & 57 \\
\hline D12 & Manhã & 22,2 & D12 & Manhã & 89 \\
\hline & Tarde & 30,4 & & Tarde & 57 \\
\hline & Noite & 30,6 & & Noite & 54 \\
\hline D13 & Manhã & 20,7 & D13 & Manhã & 79 \\
\hline & Tarde & 29,4 & & Tarde & 58 \\
\hline & Noite & 29,8 & & Noite & 61 \\
\hline D14 & Manhã & 21,6 & D14 & Manhã & 86 \\
\hline & Tarde & 30,2 & & Tarde & 61 \\
\hline & Noite & 32,4 & & Noite & 46 \\
\hline D15 & Manhã & 25 & D15 & Manhã & 84 \\
\hline & Tarde & 32,1 & & Tarde & 47 \\
\hline & Noite & 33,5 & & Noite & 35 \\
\hline D16 & Manhã & 23 & D16 & Manhã & 72 \\
\hline & Tarde & 35 & & Tarde & 32 \\
\hline & Noite & 34,2 & & Noite & 41 \\
\hline D17 & Manhã & 22,8 & D17 & Manhã & 73 \\
\hline & Tarde & 29,9 & & Tarde & 53 \\
\hline & Noite & 31,2 & & Noite & 46 \\
\hline D18 & Manhã & 21,7 & D18 & Manhã & 72 \\
\hline & Tarde & 29,3 & & Tarde & 52 \\
\hline & Noite & 34 & & Noite & 39 \\
\hline D19 & Manhã & 20,1 & D19 & Manhã & 77 \\
\hline & Tarde & 28,7 & & Tarde & 54 \\
\hline & Noite & 30,2 & & Noite & 36 \\
\hline D20 & Manhã & 19,7 & D20 & Manhã & 62 \\
\hline & Tarde & 27,8 & & Tarde & 51 \\
\hline & Noite & 31,2 & & Noite & 32 \\
\hline
\end{tabular}

cia. Nos animais antes da tosquia, nos locais com presença de lã foram realizados duas medidas uma externa e outra junto à pele após separar a lã. As mensurações das temperaturas retais e da pele foram realizadas nos mesmos momentos especificados para o exame clínico no item anterior.

Os resultados foram distribuídos em momentos, sendo que cada momento representa um intervalo de interesse, o momento 0 (M0) dos quadros corresponde às médias obtidas em vinte e dois dias de vinte animais com a lã íntegra de aproximadamente $5 \mathrm{~cm}$, os momentos 1, 2, 3 e 4 (M1, M2, M3 e M4) correspondem respectivamente aos dias 1, 2, 3 e 4, e representam os primeiros dias após a tosquia, dispostos em médias dos vinte animais tosquiados. Já os momentos 5, 6 e 7 (M5, M6 e M7) são grupamentos de dias experimentais, cada um dos momentos corresponde a cinco dias, ou seja, o M5 é um conjunto de médias dos dias 5, 6, 7, 8 e 9, e assim sucessivamente. As letras minúsculas representam a análise em coluna e as letras maiúsculas à análise em linha. As variáveis que apresentaram distribuição normal foram analisadas pela análise de variância com medidas repetidas e teste de Tukey para a comparação múltipla de médias referentes aos dois tempos (antes e pós-tosquia), momentos de avaliação e as variáveis, frequência respiratória e movimento ruminal que não apresentaram distribuição normal foram analisadas usando o teste de Mann-Whitney para comparar os grupos em cada momento e o teste de Friedman para comparar os momentos em cada grupo, seguido do teste de Dunn para comparações múltiplas. As correlações foram realizadas entre a temperatura ambiente e a umidade do ar com as outras variáveis antes e após a tosquia, assim como a temperatura retal foi correlacionada com a temperatura de superfície corporal antes e após a tosquia.

\section{RESULTADOS}

0 achado de maior relevância nos resultados da frequência cardíaca parte da diferença estatística observada no M0 em relação aos outros momentos nos períodos da tarde e da noite, e a diferença não tão marcante no período da manhã, pois neste período em alguns momentos não se observam diferenças estatísticas. Portanto, a diferença entre os animais tosquiados e os não tosquiados é marcante nos períodos da tarde e da noite, conforme elucida o Quadro 3.

A frequência respiratória declina gradativamente e tende a estabilidade a partir do dia 4 experimental. E, assim como a frequência cardíaca os animais não tosquiados apresentam valores superiores nos períodos da tarde e noite (Quadro 4).

A temperatura retal dos animais não tosquiados foi superior estatisticamente quando comparada com as temperaturas retais dos animais tosquiados em todos os mo-

Quadro 3. Média e desvio padrão da frequência cardíaca (FC) de ovinos, em bpm, antes da tosquia (M0) e pós-tosquia (M1 a M7)

\begin{tabular}{|c|c|c|c|}
\hline \multirow[t]{2}{*}{ Momento } & \multicolumn{3}{|c|}{$\bar{x} \pm s$} \\
\hline & Manhã $\left(\overline{\mathrm{x}}=31,9^{\circ} \mathrm{C}\right)$ & Tarde $\left(\overline{\mathrm{x}}=35,3^{\circ} \mathrm{C}\right)$ & Noite $\left(\overline{\mathrm{x}}=33,7^{\circ} \mathrm{C}\right)$ \\
\hline M0 & $119 \pm 7,1^{\mathrm{aA}}$ & $119 \pm 6,9^{\mathrm{aA}}$ & $119 \pm 5,8^{\mathrm{aA}}$ \\
\hline M1 & $108 \pm 11,7^{\mathrm{bcA}}$ & $90 \pm 15,7^{\mathrm{cB}}$ & $89 \pm 8,4^{\mathrm{deB}}$ \\
\hline M2 & $106 \pm 10,3^{\mathrm{bcA}}$ & $99 \pm 11,0^{\mathrm{bcAB}}$ & $92 \pm 12,2^{\mathrm{cdB}}$ \\
\hline M3 & $113 \pm 12,4^{\mathrm{abA}}$ & $97 \pm 14,0^{\mathrm{bcB}}$ & $83 \pm 7,2^{\mathrm{eC}}$ \\
\hline M4 & $103 \pm 10,0^{\mathrm{cA}}$ & $91 \pm 8,2^{\mathrm{cB}}$ & $83 \pm 9,5^{\mathrm{eC}}$ \\
\hline M5 & $94 \pm 8,0^{\mathrm{dC}}$ & $102 \pm 9,5^{\mathrm{bB}}$ & $108 \pm 7,2^{\mathrm{bA}}$ \\
\hline M6 & $111 \pm 10,4^{\mathrm{abcA}}$ & $106 \pm 10,2^{\mathrm{bB}}$ & $98 \pm 12,6^{\mathrm{cC}}$ \\
\hline M7 & $112 \pm 8,7^{\mathrm{abA}}$ & $103 \pm 9,6^{\mathrm{bB}}$ & $94 \pm 6,3^{\mathrm{cdC}}$ \\
\hline
\end{tabular}

Médias seguidas de letras distintas, minúscula na coluna e maiúscula na linha, diferem entre si pelo teste de Tukey $(\mathrm{P}<0,05)$. 
mentos experimentais e em todos os períodos. Foi possível observar que a temperatura retal foi maior durante o período da noite em relação aos outros períodos (Quadro 5).

Nos exames físicos realizados durante o experimento, não foram observadas alterações nos parâmetros de hidratação dos animais, mesmo após a tosquia. A motilidade ruminal não diferiu significativamente durante o período experimental, tanto na comparação com os animais não tosquiados quanto nos períodos, acompanhando uma variação fisiológica normal sem alterações.

A mensuração da temperatura da fronte foi realizada em local livre de lã, devido ao padrão da raça Suffolk. Neste local não ocorreu uma variação entre os momentos antes e após a tosquia, mas sim um aumento na temperatura durante o período da tarde em todos os momentos, com destaque para o M1 que apresentou uma diminuição da temperatura no período da tarde, este momento em especial reflete o primeiro dia após a tosquia dos animais, e este padrão pode ser visto em todos os outros locais de análise da temperatura da pele.

Aumento significativo da temperatura da nuca no período da tarde pode ser observado nos animais antes e após a tosquia, mas não ocorre diferença significativa entre os grupos.

A análise dos dados da temperatura externa da nuca no decorrer dos momentos apresenta uma diferença estatística que também ocorre nos dados da temperatura interna

Quadro 4. Média (x), desvio padrão (s) e mediana (Md) da frequência respiratória (FR) de ovinos, em $\mathrm{mpm}$, antes da tosquia (M0) e pós tosquia (M1 a M7)

\begin{tabular}{|c|c|c|c|c|c|c|}
\hline \multirow[t]{3}{*}{ Momento } & \multicolumn{6}{|c|}{ FR (mpm) } \\
\hline & \multicolumn{2}{|c|}{$\begin{array}{c}\text { Manhã } \\
\left(\overline{\mathrm{x}}=31,9^{\circ} \mathrm{C}\right)\end{array}$} & \multicolumn{2}{|c|}{$\begin{array}{c}\text { Tarde } \\
\left(\overline{\mathrm{x}}=35,3^{\circ} \mathrm{C}\right)\end{array}$} & \multicolumn{2}{|c|}{$\begin{array}{c}\text { Noite } \\
\left(\overline{\mathrm{x}}=33,7^{\circ} \mathrm{C}\right)\end{array}$} \\
\hline & $\bar{x} \pm s$ & Md & $\overline{\mathrm{x}} \pm \mathrm{s}$ & $\mathrm{Md}$ & $\bar{x} \pm s$ & $\mathrm{Md}$ \\
\hline M0 & $93 \pm 18,9$ & $90,0^{\mathrm{aB}}$ & $146 \pm 21,6$ & $139,5^{\mathrm{aA}}$ & $136 \pm 18,1$ & $140,0^{\mathrm{aA}}$ \\
\hline M1 & $70 \pm 15,5$ & $72,0^{\mathrm{abA}}$ & $33 \pm 9,8$ & $32,0^{\mathrm{cB}}$ & $37 \pm 8,5$ & $36,0^{\mathrm{cB}}$ \\
\hline M2 & $35 \pm 9,9$ & $32,0^{\mathrm{cB}}$ & $77 \pm 36,5$ & $72,0^{\mathrm{bA}}$ & $82 \pm 24,7$ & $76,0^{\mathrm{bA}}$ \\
\hline M3 & $90 \pm 28,0$ & $90,0^{\mathrm{aA}}$ & $113 \pm 29,0$ & $108,0^{\mathrm{abA}}$ & $38 \pm 13,6$ & $36,0^{\mathrm{cB}}$ \\
\hline M4 & $50 \pm 17,3$ & $45,0^{\mathrm{bcB}}$ & $76 \pm 25,7$ & $72,0^{\mathrm{bA}}$ & $73 \pm 23,8$ & $72,0^{\mathrm{bA}}$ \\
\hline M5 & $48 \pm 12,5$ & $45,0^{\text {bсв }}$ & $96 \pm 13,6$ & $98,5^{\mathrm{bA}}$ & $87 \pm 21,0$ & $86,0^{\mathrm{bA}}$ \\
\hline M6 & $55 \pm 13,6$ & $55,0^{\mathrm{bB}}$ & $104 \pm 25,3$ & $97,5^{\mathrm{bA}}$ & $96 \pm 23,0$ & $94,5^{\text {abA }}$ \\
\hline M7 & $49 \pm 10,1$ & $46,0^{\mathrm{bcB}}$ & $92 \pm 19,1$ & $94,0^{\mathrm{bA}}$ & $92 \pm 20,9$ & $92,5^{\mathrm{bA}}$ \\
\hline
\end{tabular}

Medianas seguidas de letras distintas, minúscula na coluna e maiúscula na linha, diferem entre si pelo teste de Dunn $(\mathrm{P}<0,05)$.

Quadro 5. Média ( $\bar{x})$ e desvio padrão (s) da temperatura retal (TR) em ovinos, $\mathrm{em}^{\circ} \mathrm{C}$, antes da tosquia (M0) e pós tosquia (M1 a M7)

\begin{tabular}{|c|c|c|c|}
\hline \multirow[t]{2}{*}{ Momento } & \multicolumn{3}{|c|}{$\bar{x} \pm s$} \\
\hline & Manhã $\left(\overline{\mathrm{x}}=31,9^{\circ} \mathrm{C}\right)$ & Tarde $\left(\bar{x}=35,3^{\circ} \mathrm{C}\right)$ & Noite $\left(\bar{x}=33,7^{\circ} \mathrm{C}\right)$ \\
\hline M0 & $39,4 \pm 0,19 \mathrm{aC}$ & $39,8 \pm 0,18^{\mathrm{aB}}$ & $39,9 \pm 0,22^{\mathrm{aA}}$ \\
\hline M1 & $39,0 \pm 0,25^{b A}$ & $38,7 \pm 0,17^{\mathrm{cB}}$ & $38,9 \pm 0,35^{\mathrm{dA}}$ \\
\hline M2 & $38,9 \pm 0,44^{\mathrm{bB}}$ & $39,0 \pm 0,21^{\mathrm{bB}}$ & $39,3 \pm 0,30^{\mathrm{bA}}$ \\
\hline M3 & $38,9 \pm 0,28^{\mathrm{bA}}$ & $38,9 \pm 0,27^{\mathrm{bA}}$ & $38,5 \pm 0,25^{\mathrm{eB}}$ \\
\hline M4 & $38,9 \pm 0,30^{\mathrm{bA}}$ & $38,9 \pm 0,44^{\mathrm{bA}}$ & $39,0 \pm 0,26^{\mathrm{cdA}}$ \\
\hline M5 & $38,6 \pm 0,26^{c C}$ & $39,0 \pm 0,18^{\mathrm{bB}}$ & $39,3 \pm 0,19^{\mathrm{bA}}$ \\
\hline M6 & $38,9 \pm 0,19^{\mathrm{bcc}}$ & $39,0 \pm 0,23^{\mathrm{bB}}$ & $39,1 \pm 0,20^{\mathrm{bcA}}$ \\
\hline M7 & $38,8 \pm 0,13^{\mathrm{bcB}}$ & $38,9 \pm 0,22^{\mathrm{bB}}$ & $39,1 \pm 0,18^{\operatorname{cdA}}$ \\
\hline
\end{tabular}

Médias seguidas de letras distintas, minúscula na coluna e maiúscula na linha, diferem entre si pelo teste de Tukey $(\mathrm{P}<0,05)$. da nuca onde as temperaturas do M0 são muito superiores aos outros momentos, ou seja, antes da tosquia a temperatura da nuca foi estatisticamente superior as temperaturas após a tosquia e mostra que a temperatura externa tem maior correlação $(r=0,68, p<0,05)$ com a temperatura da pele sem lã quando comparada com a temperatura interna.

Na região do dorso foram relatadas as maiores temperaturas quando comparados com os outros locais analisados. Os períodos da manhã e tarde, no M0 da temperatura externa do dorso, apresentaram as menores temperaturas quando comparadas às temperaturas do dorso do grupo tosquiado, o contrário é observado no M0 no período da tarde, onde ocorre diferença estatística maior em relação aos outros momentos deste período. A temperatura interna do dorso apresentou diferença significativa maior em relação aos outros momentos e em todos os períodos no M0, ou seja, antes da tosquia as temperaturas deste ponto foram superiores às temperaturas do dorso após a tosquia.

A temperatura externa da face externa da coxa foi menor nos animais antes da tosquia, mas a temperatura interna da face externa da coxa quando comparada com os pontos da face externa da coxa do após tosquia foi estatisticamente significativa, sendo maior a temperatura antes da tosquia.

As temperaturas do períneo dos animais antes da tosquia apresentaram diferença estatística significativa quando comparadas as temperatura após tosquia e a partir do M4 ocorre estabilização das temperaturas.

Após a tosquia os animais apresentaram uma queda na temperatura da axila no primeiro dia após a tosquia. As temperaturas do grupo não tosquiado apresentam diferença estatística significativa quando comparadas às temperaturas após tosquia no decorrer dos momentos e em todos os períodos, principalmente nos períodos da tarde e noite, e a partir do M4 tende a não mais variar estatisticamente entre os dois tempos (antes e pós-tosquia).

Os animais examinados após tosquia apresentaram uma queda na temperatura da face interna da coxa no primeiro dia após a tosquia, como foi observado nos outros locais de análise. As temperaturas dos animais após a tosquia apresentaram diferença estatística significativa quando comparadas às temperaturas dos ovinos após a tosquia no decorrer dos momentos e em todos os períodos, principalmente nos períodos da tarde e noite, e a partir do M4 a variação tende a não variar estatisticamente.

A correlação entre a temperatura ambiente e as variáveis dos animais antes da tosquia apresentou alguns resultados significativos com valores negativos. As temperaturas da superfície corpórea nos períodos da manhã e da tarde apresentaram correlação positiva, ou seja, quando a temperatura ambiente aumenta durante o dia os pontos analisados aumentam (Quadro 6).

Após a tosquia dos animais, quando comparamos a temperatura ambiente às outras variáveis fisiológicas medidas, notamos que os dados da frequência respiratória se tornam, após a tosquia, significativos e com valores positivos, ou seja, quando a temperatura ambiente aumenta, aumenta também a frequência respiratória. E todos os dados das temperaturas da pele são significativos, com exceção das temperaturas do períneo e axila no período da noite, mostrando que após 
Quadro 6. Coeficiente de correlação entre a temperatura ambiente, $\mathrm{em}^{\circ} \mathrm{C}$, e os parâmetros e temperaturas de ovinos,

freqüência cardíaca (FC), em bpm, frequência respiratória (FR), em mpm, movimento ruminal (MR), em mpm, temperatura retal (TR), em ${ }^{\circ} \mathrm{C}$, temperatura da fronte (TF), em ${ }^{\circ} \mathrm{C}$, temperatura externa da nuca (TEN), $\mathrm{em}^{\circ} \mathrm{C}$, temperatura interna da nuca (TIN), em ${ }^{\circ} \mathrm{C}$, temperatura externa do dorso (TED), em ${ }^{\circ} \mathrm{C}$, temperatura interna do dorso (TID), $\mathrm{em}^{\circ} \mathrm{C}$, temperatura externa da face externa da coxa (TEFEC), em ${ }^{\circ} \mathrm{C}$, temperatura interna da face externa da coxa (TIFEC), $\mathrm{em}^{\circ} \mathrm{C}$, temperatura períneo (TP), em ${ }^{\circ} \mathrm{C}$, temperatura da axila (TA), em ${ }^{\circ} \mathrm{C}$, temperatura da face interna da coxa (TFIC), $\mathrm{em}^{\circ} \mathrm{C}$, com os dados do termômetros FLUKE $^{\circledR}$ 66, dos animais antes da tosquia, segundo os períodos de avaliação

\begin{tabular}{lccc}
\hline Variável & Manhã $\left(\overline{\mathrm{x}}=31,9^{\circ} \mathrm{C}\right)$ & Tarde $\left(\overline{\mathrm{x}}=35,3^{\circ} \mathrm{C}\right)$ & Noite $\left(\overline{\mathrm{x}}=33,7^{\circ} \mathrm{C}\right)$ \\
\hline FC & $-0,19$ & $-0,46^{*}$ & $-0,44^{*}$ \\
FR & $-0,08$ & $-0,10$ & $-0,20$ \\
MR & $-0,07$ & $-0,12$ & $-0,44^{*}$ \\
TR & $-0,27$ & $-0,51^{*}$ & $-0,50^{*}$ \\
TF & $-0,40$ & $-0,52^{*}$ & $-0,18$ \\
TEN & $-0,71^{*}$ & $-0,48^{*}$ & $-0,21$ \\
TIN & $-0,33$ & $-0,40$ & $-0,14$ \\
TED & $-0,47^{*}$ & $-0,51^{*}$ & $-0,23$ \\
TID & $-0,36$ & $-0,50^{*}$ & $-0,21$ \\
TEFEC & $-0,43^{*}$ & $-0,47^{*}$ & $-0,26$ \\
TIFEC & $-0,44^{*}$ & $-0,37$ & $-0,22$ \\
TP & $-0,43^{*}$ & $-0,49^{*}$ & $-0,44^{*}$ \\
TA & $-0,35$ & $-0,62^{*}$ & $-0,63^{*}$ \\
TFIC & $-0,54^{*}$ & $-0,67^{*}$ & $-0,59^{*}$
\end{tabular}

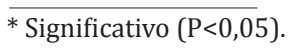

Quadro 7. Coeficiente de correlação entre a temperatura ambiente, em ${ }^{\circ} \mathrm{C}$, e os parâmetros e temperaturas de ovinos, frequência cardíaca (FC), em bpm, frequência respiratória

(FR), em mpm, movimento ruminal (MR), em mpm, temperatura retal (TR), em ${ }^{\circ} \mathrm{C}$, temperatura da fronte (TF), em ${ }^{\circ} \mathrm{C}$, temperatura da nuca (TN), em ${ }^{\circ} \mathrm{C}$, temperatura do dorso (TD), $\mathrm{em}^{\circ} \mathrm{C}$, temperatura da face externa da coxa (TFEC), em ${ }^{\circ} \mathrm{C}$, temperatura períneo (TP), em ${ }^{\circ} \mathrm{C}$, temperatura da axila (TA), $\mathrm{em}^{\circ} \mathrm{C}$, temperatura da face interna da coxa (TFIC), em ${ }^{\circ} \mathrm{C}$, com os dados do termômetro FLUKE ${ }^{\circledR}$ 66, dos animais após a tosquia, segundo os períodos de avaliação

\begin{tabular}{lccc}
\hline Variável & Manhã $\left(\overline{\mathrm{x}}=31,9^{\circ} \mathrm{C}\right)$ & Tarde $\left(\overline{\mathrm{x}}=35,3^{\circ} \mathrm{C}\right)$ & Noite $\left(\overline{\mathrm{x}}=33,7^{\circ} \mathrm{C}\right)$ \\
\hline FC & $-0,19$ & $-0,16$ & $-0,11$ \\
FR & $0,69^{*}$ & $0,63^{*}$ & $0,64^{*}$ \\
MR & $-0,24$ & $-0,24$ & $-0,31$ \\
TR & 0,05 & 0,34 & 0,20 \\
TF & $0,81^{*}$ & $0,89^{*}$ & $0,84^{*}$ \\
TN & $0,78^{*}$ & $0,87^{*}$ & $0,85^{*}$ \\
TD & $0,71^{*}$ & $0,84^{*}$ & $0,67^{*}$ \\
TFEC & $0,77^{*}$ & $0,89^{*}$ & $0,80^{*}$ \\
TP & $0,75^{*}$ & $0,72^{*}$ & 0,41 \\
TA & $0,64^{*}$ & $0,62^{*}$ & 0,38 \\
TFIC & $0,63^{*}$ & $0,69^{*}$ & $0,57^{*}$
\end{tabular}

$\bar{*}$ Significativo $(\mathrm{P}<0,05)$.

a tosquia os dados passam a acompanhar positivamente as variações da temperatura ambiente (Quadro 7).

A correlação da umidade ambiente com as outras variáveis dos animais antes da tosquia foi negativa ou muito próxima de zero, assim como os dados da temperatura ambiente. A frequência respiratória neste ponto difere, pois ela é significativa em todos os períodos, sendo menor quanto mais alta for a umidade do ar. Basicamente todos os dados das temperaturas da pele apresentaram correlação negativa nos períodos da tarde e noite.

A frequência respiratória permaneceu com correlação significativa e negativa após a tosquia. 0 período da manhã obteve correlação significativa e positiva para as temperaturas da pele. 0 contrário ocorreu nos períodos da tarde e noite. Dessa forma, observou-se que durante a manhã as temperaturas da pele acompanham as variações da umidade do ar, e durante a tarde e noite as temperaturas acompanham negativamente as variações da umidade do ar.

A correlação entre a temperatura retal e as temperaturas da superfície da pele foram significativas e em sua maioria altas, ou seja, antes da tosquia, a temperatura da pele acompanha a temperatura retal (Quadro 8).

Após a tosquia, somente a temperatura do períneo no período da tarde e a temperatura da face interna da coxa nos períodos da tarde e noite, obtiveram alguns pontos significantes estatisticamente, todas as outras temperaturas não se correlacionam significativamente com a temperatura retal. Ocorreu, após a tosquia, uma mudança na associação das temperaturas, as temperaturas da pele não mais acompanharam a temperatura retal (Quadro 9).

Quadro 8. Coeficiente de correlação entre a temperatura retal, em ${ }^{\circ} \mathrm{C}$, e as temperaturas da fronte (TF), em ${ }^{\circ} \mathrm{C}$, externa da nuca (TEN), em ${ }^{\circ} \mathrm{C}$, interna da nuca (TIN), em ${ }^{\circ} \mathrm{C}$, externa do dorso (TED), em ${ }^{\circ} \mathrm{C}$, interna do dorso (TID), em ${ }^{\circ} \mathrm{C}$, externa da face externa da coxa (TEFEC), $\mathrm{em}^{\circ} \mathrm{C}$, interna da face externa da coxa (TIFEC), em ${ }^{\circ} \mathrm{C}$, períneo (TP), em ${ }^{\circ} \mathrm{C}$, da axila (TA), $\mathrm{em}^{\circ} \mathrm{C}$, da face interna da coxa (TFIC), em ${ }^{\circ} \mathrm{C}$, com os dados do termômetro FLUKE $^{\circledR} 66$, dos animais antes da tosquia, segundo os períodos de avaliação

\begin{tabular}{lccc}
\hline Variável & Manhã $\left(\overline{\mathrm{x}}=31,9^{\circ} \mathrm{C}\right)$ & Tarde $\left(\overline{\mathrm{x}}=35,3^{\circ} \mathrm{C}\right)$ & Noite $\left(\overline{\mathrm{x}}=33,7^{\circ} \mathrm{C}\right)$ \\
\hline TF & $0,56^{*}$ & $0,76^{*}$ & $0,79^{*}$ \\
TEN & 0,35 & $0,76^{*}$ & $0,77^{*}$ \\
TIN & 0,31 & $0,69^{*}$ & $0,75^{*}$ \\
TED & $0,54^{*}$ & $0,76^{*}$ & $0,79^{*}$ \\
TID & $0,56^{*}$ & $0,67^{*}$ & $0,79^{*}$ \\
TEFEC & $0,65^{*}$ & $0,66^{*}$ & $0,79^{*}$ \\
TIFEC & $0,59^{*}$ & $0,56^{*}$ & 0,43 \\
TP & $0,50^{*}$ & $0,84^{*}$ & $0,83^{*}$ \\
TA & 0,42 & $0,83^{*}$ & $0,89^{*}$ \\
TFIC & $0,61^{*}$ & $0,79^{*}$ & $0,88^{*}$ \\
* Significativo $(\mathrm{P}<0,05)$. & &
\end{tabular}

Quadro 9. Coeficiente de correlação entre a temperatura retal, em ${ }^{\circ} \mathrm{C}$, e as variáveis temperaturas da fronte (TF), em ${ }^{\circ} \mathrm{C}$, temperatura da nuca (TN), $\mathrm{em}^{\circ} \mathrm{C}$, temperatura do dorso (TD), $\mathrm{em}^{\circ} \mathrm{C}$, temperatura da face externa da coxa (TFEC), em ${ }^{\circ} \mathrm{C}$, temperatura períneo (TP), em ${ }^{\circ} \mathrm{C}$, temperatura da axila (TA), em ${ }^{\circ} \mathrm{C}$, temperatura da face interna da coxa (TFIC), em ${ }^{\circ} \mathrm{C}$, com os dados do termômetro $\mathrm{FLUKE}^{\circledR}$, dos animais após a tosquia, segundo os períodos de avaliação

\begin{tabular}{lccc}
\hline Variável & Manhã $\left(\overline{\mathrm{x}}=31,9^{\circ} \mathrm{C}\right)$ & Tarde $\left(\overline{\mathrm{x}}=35,3^{\circ} \mathrm{C}\right)$ & Noite $\left(\overline{\mathrm{x}}=33,7^{\circ} \mathrm{C}\right)$ \\
\hline TF $^{1}$ & 0,32 & 0,41 & 0,23 \\
TN $^{1}$ & 0,22 & 0,31 & 0,23 \\
TD $^{1}$ & 0,18 & 0,38 & 0,19 \\
TFEC $^{1}$ & 0,23 & 0,32 & 0,29 \\
TP $^{1}$ & 0,40 & 0,44 & $0,54^{*}$ \\
TA $^{1}$ & 0,31 & 0,44 & 0,16 \\
TFIC $^{1}$ & 0,36 & $0,53^{*}$ & $0,47^{*}$
\end{tabular}

$\bar{*}$ Significativo $(\mathrm{P}<0,05)$. 


\section{DISCUSSÃO}

O efeito do estresse térmico após a tosquia mostrou-se evidente nos animais testados, refletindo alterações marcantes nos parâmetros frequência cardíaca, frequência respiratória e temperatura retal, resultados também descritos por Klemm (1961), Eyal (1963), Robinson (1969), Avondo et al. (2000), Ortêncio Filho et al. (2001), Piccione et al. (2002), Piccione \& Caola (2003) e Marai et al. (2008). A temperatura da superfície da pele também variou após a tosquia, mas de forma a diminuir as temperaturas de superfície.

Antes da tosquia os animais apresentaram freqüência respiratória estabelecida como a mais confiável dos parâmetros para estabelecer um quadro de estresse térmico, com valores em média três vezes maiores que os descritos por Gonçalves \& Feitosa (2008), que descrevem como fisiológica a frequência entre 20 e 30 movimentos por minuto em animais adultos. A exposição em ambientes de altas temperaturas promove gasto de energia para dissipar o calor, resultando em aumento da frequência respiratória, temperatura corpórea e consumo de água e declínio na ingestão alimentar.

0 estresse térmico refletido na frequência respiratória também é descrito por Marai et al. (2008) e também é exposto como parâmetro com variação significativa após a tosquia por Klemm (1961), Eyal (1962), Robinson (1969) e Hopkins (1978). Somado ao fato de obter correlação positiva com a temperatura ambiente.

Quando a freqüência respiratória foi correlacionada com a umidade relativa do ar, passou a ser inversamente proporcional. Robertshaw (2006) relata a perda de calor pelas vias respiratórias nos ovinos, e essa perda é acentuada em um ambiente com umidade do ar favorável, pois o resfriamento ocorre não só pela troca de ar, mas também pelo resfriamento do espaço morto e consequente resfriamento do sangue da região. Além disso, quanto maior for a umidade do ar menor é a capacidade de termorregulação por evaporação, e por isso a perda de calor pela respiração passa a ter maior importância.

A frequência cardíaca acompanha a variação térmica ambiente e também é indicador de estresse térmico. Nos períodos da tarde e noite são encontradas as maiores variações entre o grupo tosquiado e não tosquiado, confirmando o quadro de estresse nos animais com lã. 0 aumento da frequência cardíaca ocorre em resposta à necessidade de transporte vascular de oxigênio e substrato energético para suprir o aumento da frequência respiratória na tentativa de compensação térmica com a perda por troca gasosa, somando ao gasto de energia para dissipar o calor (Marai et al. 2008).

Os valores maiores de temperaturas e parâmetros dos animais antes da tosquia permaneceram durante todo o experimento demonstrando que os valores descritos em literatura não são aplicáveis aos animais submetidos a este tipo de clima. Esta variação mostra uma adaptabilidade dos animais da raça Suffolk com as regiões de clima quente, mas não significa que animais em conforto térmico não apresentem melhor rendimento.

Apesar de estudos relatarem aumento na ingestão de alimentos e consequente aumento dos movimentos ru- minais após a tosquia (Costa et al. 1992, Ortêncio Filho et al. 2001, Marai et al, 2008), no presente estudo não foram significativos os aumentos de movimentos ruminais após a tosquia.

O estado de hidratação dos animais por avaliação clínica não alterou antes ou após a tosquia, não gerando dados que justificassem a análise estatística, mesmo com relatos de alteração da evaporação cutânea maior em animais tosquiados (Piccione et al. 2002, Silva \& Starling 2003). Quando expostos à um ambiente quente ocorre uma variação na temperatura retal e da pele e a quantidade de água perdida pela transpiração pode ser maior em animais tosquiados, mas estes fatores não influem na avaliação clínica do equilíbrio hídrico dos animais.

O grupo tosquiado apresentou a temperatura retal até $1,4^{\circ} \mathrm{C}$ menor que o grupo não tosquiado. Essa diferença se torna maior no período da noite devido a presença da lã funcionando como uma camada isolante, este efeito garante que a temperatura dentro do velo sofra trocas mais lentas de calor com o ambiente, e garante a variação entre os dois tempos (antes e após tosquia) mesmo no período da noite, pois o calor armazenado no período da tarde ainda não foi dissipado para o ambiente. Os resultados encontrados no presente estudo não condizem com os achados de Piccione et al. $(2002,2003)$ e Hopkins et al. (1978), onde ocorreu um aumento da temperatura retal após a tosquia de até $1^{\circ} \mathrm{C}$, mas lembrando que estes estudos foram conduzidos em países com clima mediterrâneo onde a temperatura ambiente oscila entre 16 e $28^{\circ} \mathrm{C}$ e que no presente estudo, tal temperatura oscilou entre 20 e $53^{\circ} \mathrm{C}$.

Em todas as tabelas de correlação entre as temperaturas da pele e os grupos, o M1 apresenta valores de correlação menores, este fato é explicado pelo infortúnio climático neste dia em específico. Enquanto a média das temperaturas ambiente foi de $27,6^{\circ} \mathrm{C}$, o M1 não apresentou temperatura ambiente maior que $24^{\circ} \mathrm{C}$.

As temperaturas obtidas da face externa da coxa não são confiáveis, pois a localização é prejudicada pelos hábitos das ovelhas de deitar sobre a coxa, promovendo a troca de temperatura por condução com o solo, além de muitas vezes encontrar o local umedecido no período da manhã, pela maior umidade presente na superfície do solo.

A temperatura da fronte foi o único ponto deslanado que sofre interferência direta do sol, já que os animais são mantidos a pasto, sendo assim, mesmo as coletas de dados sendo feitas em local coberta, os animais ficam submetidos todo dia ao sol. Neste ponto a temperatura é superior no grupo não tosquiado nos períodos da tarde e da noite, mas no grupo tosquiado existem momentos que não diferem significativamente do grupo não tosquiado e como um todo este ponto parece só sofrer interferência da temperatura ambiente e da temperatura corpórea e não da lã.

Com exceção dos pontos externos da coxa e fronte, os outros pontos podem ser divididos em três padrões, o primeiro com variação significativa entre os grupos tosquiados e não tosquiado apenas no período da tarde, onde cabem os pontos externo da nuca e externo do dorso. $\mathrm{O}$ segundo com variação significativa no período da tarde e noite, nos pontos interno da nuca, períneo, axila e face interna da coxa e 
o terceiro padrão envolvendo o ponto interno do dorso que apresenta variação significativa nos três períodos podendo afirmar que os locais onde há incidência solar direta são os locais onde a variação é maior entre os grupos, assim como períodos de maior incidência solar são mais afetados pelo efeito da lã.

Os pontos que mais se correlacionaram com a temperatura retal foram os da temperatura do períneo, axila e face interna da coxa, ou seja, quando a temperatura a temperatura retal aumenta estes pontos acompanham esta variação com uma maior fidelidade.

As correlações entre as temperaturas da superfície corpórea com a temperatura ambiente e umidade do ar apresentaram resultados negativos, explicados pelo efeito isolante da lã, ou seja, mesmo com um aumento da temperatura e umidade do ar, a temperatura corpórea tende a manter um equilíbrio compensatório. Portanto, os animais não tosquiados apresentam uma temperatura retal elevada, com aumento de temperatura de superfície, e tentativa de compensação pela troca de calor na respiração, mas a lã impede que ocorra a variação segundo a temperatura ambiente (Piccione et al. 2002, 2003).

Os resultados obtidos da correlação entre a temperatura da pele com a temperatura ambiente e umidade o ar obtidos após a tosquia, são contrários aos observados nos animais não tosquiados, a correlação além de positiva é significativa, o que demonstra que a temperatura da pele aumenta quando a temperatura ambiente aumenta. Esse fato é explicado simplesmente pela retirada da camada isolante que possui uma baixa condutividade térmica (Aleksiev et al. 2003).

A umidade relativa do ar não demonstrou ser um fator agravante no processo de tosquia, pois os dados obtidos antes e após a tosquia são muito semelhantes e de baixa significância.

$\mathrm{Na}$ análise da variação da temperatura retal foi possível afirmar que ocorre uma variação após a tosquia, com diminuição da temperatura, mas além destes resultados a correlação entre a temperatura retal e as temperaturas da superfície da pele também agrega resultados importantes. Antes da tosquia as temperaturas dos pontos da superfície da pele acompanham a variação da temperatura retal, mas após a tosquia essa variação não mais ocorre, ou seja, após a tosquia quando a temperatura retal aumenta a temperatura da pele não acompanha a variação.

A temperatura retal é usada freqüentemente como índice de adaptabilidade fisiológica aos ambientes quentes, pois seu aumento mostra que os mecanismos de perda de calor tornaram-se insuficientes para manter a homeotermia (Johnson 1980, Mota 1997, Pereira 2008). Já a freqüência respiratória é um indicador mais sensível do estresse calórico do que a temperatura retal (Brown-Brandl et al., 2005). Sob estresse térmico, para manutenção da temperatura corporal há também aumento da temperatura de superfície corporal e freqüência respiratória, que começam a elevar-se antes da temperatura retal (Bianca 1965, Fereira et al., 2006).

A perda por transpiração nos animais não tosquiados é diminuída e somando ao fator de sobrecarga térmica no in- terior do velo temos uma troca térmica deficiente, ou seja, a perda de calor não é suficiente. Possivelmente o que ocorre é o retorno da eficiência da troca de calor com o ambiente após a tosquia, sem a presença do velo a capacidade de perda por transpiração aumenta levando os animais a um estado de compensação térmica positiva (Piccione et al. 2002).

\section{CONCLUSÕES}

Ocorre após a tosquia uma variação na temperatura corpórea dos ovinos lanados de regiões quentes e secas. Essa variação, contradizendo trabalhos realizados na Europa, mostra que a temperatura diminui após a retirada do velo, explicada pela ausência da camada isolante, que interfere na perda de calor com o ambiente.

Neste estudo os melhores indicadores de estresse térmico e, portanto as variáveis clínicas mais afetadas pelo aumento da temperatura e pela tosquia são a frequência respiratória, a temperatura retal e as temperaturas da superfície da pele, entre elas a temperatura do períneo, axila e face interna da coxa são as mais confiáveis.

Os parâmetros avaliados demonstram que o conforto térmico dos ovinos mantidos em condições quentes e secas foi alcançado, sendo que os animais utilizados encontram-se adaptados ao ambiente.

\section{REFERÊNCIAS}

Aleksiev I., Gudev D., Popova-Ralcheva S. \& Moneva P. 2003. Thermoregulation in sheep.II. Dynamics of rectal temperature before and after shearing of sheep exposed to sun or kept in shade. Zhivotnov' dni-Nauki 40:32-36.

Anderson B.E. 1996. Regulação da temperatura e fisiologia ambiental, p.805-813. In: Swenson M.J. (Ed.), Dukes Fisiologia dos Animais Domésticos. $11^{\mathrm{a}}$ ed. Guanabara, Rio de Janeiro.

Avondo M., Bordonaro S., Marletta D., Guastella A.M. \& D’Urso G. 2000. Effects of shearing and supplemental level on intake of dry ewes grazing on barley stubble. Small Rumin. Res. 38:237-241.

Carvalho E.B., Oliveira M.A.G. \& Domingues P.F. 2004. Base para a Criação de Ovinos no Estado de São Paulo. 3a ed. ASPACO, São Manuel. 92p.

Çam M.A., Olfaz M. \& Garipoglu A.V. 2007. Shearing male lambs in the cold season improves the carcass yield without affecting fattening performance. Anim. Sci. J. 78:259-265.

Eyal E. 1963. Shorn and unshorn Awassi sheep III. Respiration rate. J. Agricult. Sci. 60:75-181.

Filho H.O., Barbosa O.R., Sakaguti E.S., Onorato W.M. \& Macedo F.A.F. 2001. Efeito da tosquia sobre o comportamento de ovelhas das raças Texel e Hampshire Down, ao longo do período noturno, no Noroeste do Estado do Paraná. Acta Scientiarum 23:995-1001.

Golçalves R.C. \& Feitosa F.L.F. 2008. Semiologia do sistema respiratório de grandes animais, p.275-292. In: Feitosa F.L.F. (Ed.), Semiologia Veterinária: a arte do diagnóstico. $2^{\underline{a}}$ ed. Roca, São Paulo.

Hershler C., Conine T.A., Numm A. \& Hannay M. 1992. Assessment of an infra-red non-contact sensor for routine skin temperature monitoring: a preliminary study. J. Med. Eng. Technol. 16:117-122.

Hopkins P.S., Knights G.I. \& Feuvre A.S. 1978. Studies of the environmental physiology of tropical merinos. Aust. J. Agricult. Res. 29:161-171.

Klemm G.H. 1962. The reactions of unshorn and shorn sheep to hot wet and dry atmospheres. Aust. J. Agricult. Res. 13:472-478.

Laloni L.A., Naas I.A., Macari M., Pinheiro M.G. \& Pereira D.F. 2002. Avaliação da relação entre temperatura da superfície corporal e produção de vacas jersey submetidas á climatização na pré-ordenha. Ciênc. Engenharia 11:35-37.

Marai L.F.M., El-Darawany A.A., Fadiel A. \& Abdel-Hafez M.A.M. 2008. Re- 
productive performance traits as affected by heat stress and its alleviation in sheep. Tropical and Subtropical Agroecosystems 8:209-234.

Matsukuma B.H., Peiró J.R., Feitosa F.L.F. \& Mendes L.C.N. 2010. Fisiologia da termorregulação e estresse térmico em ovinos lanados. Revta CFMV 16:26-30.

Ortêncio Filho H., Barbosa O.R., Sakaguti E.S., Onorato W.M. \& Macedo F.A.F. 2001. Efeito da tosquia sobre o comportamento de ovelhas das raças Texel e Hampshire Down, ao longo do período noturno, no Noroeste do Estado do Paraná. Acta Scientiarum 23:995-1001.

Piccione G. \& Caola G. 2003. Influence of shearing on the circadian rhythm of body temperature in the sheep. J. Vet. Med. A 50:235-240.

Piccione G., Caola G. \& Refinetti R. 2002. Effect of shearing on the core body temperature of three breeds of Mediterranean sheep. Small Rumin. Res. 46:211-215.
Quesada M., McManus C. \& Couto F.A.D. 2001. Tolerância ao calor de duas raças de ovinos deslanados no distrito federal. Revta Bras. Zootec. 30:1021-1026.

Robertshaw D. 2006. Regulação da temperatura e o ambiente térmico, p.897-905. In: Swenson M.J. (Ed.), Dukes' Fisiologia dos Animais Domésticos. $12^{\mathrm{a}}$ ed. Guanabara, Rio de Janeiro.

Robinson D.W. 1969. Preliminary observations on the heat tolerance of shorn and nutritionally depleted sheep in a tropical environment. Brit. Vet. J. 125:112-125.

Saegusa Y. \& Tabata H. 2003. Usefulness of infrared thermometry in determining body temperature in mice. Lab. Anim. Sci. 65:1365-1367.

Silva R.G. \& Starling J.M.C. 2003. Evaporação cutânea e respiratória em ovinos sob altas temperaturas ambientes. Revta Bras. Zootec. 32:19561961. 